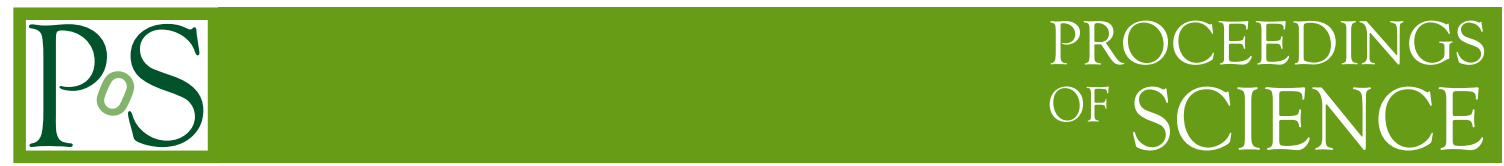

\title{
Coherent light-matter interactions
}

\author{
Jean-Louis Le Gouët* \\ Laboratoire Aimé Cotton, bâtiment 505, campus universitaire, 91405 Orsay cedex, France \\ E-mail: jean-louis.legouet@lac.u-psud.fr
}

\begin{abstract}
We consider the propagation of a weak plane-wave classical coherent pulse through an ensemble of absorbing atoms. This elementary problem offers an ideal playground to discuss causality, atomic superposition state, various atomic relaxation processes, and exchange of quantum information between light and matter. This very simple introductory presentation pays special attention to the connection between time and frequency domains. Features such as slow light can be derived in this basic framework.
\end{abstract}

Quantum of Quasars workshop

December 2-4, 2009

Grenoble, France

* Speaker. 


\section{Introduction}

The blackbody radiation is the most common expression of light in astrophysics. Classical electrodynamics offers the usual framework to describe thermal radiation, but covers a much wider range of electromagnetic phenomena. The scope of classical electrodynamics also spills over into quantum physics. This specifically occurs in the semi-classical theory of light-atom interaction, where fully quantum mechanical atoms interact with classical light. The analysis of emission and absorption spectral lines, as observed in astrophysics, is based on such a description. Those spectral lines represent the electromagnetic manifestation of atomic superposition states.

Whether thermal or not, the radiation observed in astrophysics is usually incoherent, with a duration much larger than the inverse bandwidth. In the present paper we focus on the opposite situation of totally coherent pulses, whose duration equals the inverse spectral width ${ }^{1}$. Although it is probably unlikely that such radiation could be observed in astrophysics, the simplicity and richness of the corresponding physics make it worth considering.

We discuss the most elementary situation of a weak plane-wave coherent pulse propagating through an absorbing ensemble of two-level atoms. All the atoms are assumed to be initially sitting in their ground state. With this extremely simple system we explore such features as causality, pulse area propagation, and slow light.

\section{Causality and refraction index}

Attenuation of light by an absorbing material is governed by the well known law of Beer Lambert. According to this law, the power spectrum $\left|A_{\text {in }}(\omega)\right|^{2}$ of the incoming weak field undergoes an exponential attenuation as a function of the propagation depth $z$ :

$$
\left|A_{\text {out }}(\omega)\right|^{2}=\left|A_{\text {in }}(\omega)\right|^{2} \exp (-\alpha(\omega) z)
$$

The names of August Beer (1825-1863) and Johann Heinrich Lambert (1728-1777) are attached to this law, but its origin can be traced back to the Essai sur la gradation de la lumière that Pierre Bouguer published in 1729. When the incoming pulse spectrum is much narrower than the absorption band, this law also applies to the field amplitude, which leads to:

$$
A_{\text {out }}(\omega)=A_{\text {in }}(\omega) \exp (-\alpha(\omega) z / 2)
$$

Provided $\alpha(\omega)$ does not significantly vary over the pulse spectrum, the pulse is only attenuated, preserving its temporal shape as it propagates through the absorbing medium.

This is no longer true when the pulse spectrum is much broader than the absorption band. To address the problem in the simplest way, let us first consider a small optical density material. Then Eq. 2.2 can be expanded as:

$$
A_{\text {out }}(\omega)=A_{\text {in }}(\omega)-A_{\text {in }}(\omega) \alpha(\omega) z / 2
$$

\footnotetext{
${ }^{1}$ to be specific, a black body coherent pulse, with a spectrum centered in the visible, would last for $\approx 1$ femtosecond. Observing the continuous black body emission with an ultrafast detector would not help to discriminate the propagation features we consider in this paper. Indeed, in standard conditions, those features can only be observed on a dark background, just after the extinction of a minimum-duration incoming pulse. A coherent pulse, with a duration given by the inverse bandwidth of the emitted light, is the shortest conceivable emission limited to a given bandwidth.
} 
The first term on the right-hand side coincides with the incoming pulse. In the second one, $A_{\text {in }}(\omega)$ is filtered by the absorption band profile $\alpha(\omega)$, whose spectral width is denoted $\Delta_{0}$. In the time domain, this corresponds to a pedestal spreading over a time interval of order $1 / \Delta_{0}$, much larger than the pulse duration. Hence the pedestal starts before the arrival of the pulse. This clearly violates causality and invalidates Eq. 2.2. We have incorrectly described the atomic response to the radiation field. In the weak field limit, the atomic response is certainly linear. However, this does not mean that the macroscopic polarization at time $t$ is just proportional to the local field at the same time. The atomic response is not instantaneous. Instead it keeps some memory of interactions with the field at previous times. More precisely, the response at time $t$ results from the linear combination of all the elementary linear responses to the successive interactions with the field at any time before $t$. Following this intuitive approach, one can express the macroscopic polarization as:

$$
P(z, t)=\varepsilon_{0} \int_{-\infty}^{\infty} R\left(t-t^{\prime}\right) A\left(z, t^{\prime}\right) d t^{\prime}
$$

where the response function $R\left(t-t^{\prime}\right)$ just accounts for the evolution of the atomic system from the interaction time $t^{\prime}$, up to the observation time $t$. Since the integration in Eq. 2.4 runs from $-\infty$ to $+\infty$, the response function must satisfy the causality condition:

$$
R\left(t-t^{\prime}\right)=\theta\left(t-t^{\prime}\right) R\left(t-t^{\prime}\right)
$$

where $\theta(t)$ stands for the unit step function. The finite width of $R(t)$ reflects the finite width of the absorption band and the finite lifetime of the atomic superposition states. A $\Delta_{0}$-bandwidth means that this lifetime exceeds $1 / \Delta_{0}$. By time-to-frequency Fourier transformation of Eq. 2.4, one recovers the well known linear expression of the macroscopic polarization in terms of the electric field.

$$
\tilde{P}(z, \omega)=\varepsilon_{0} \tilde{R}(\omega) \tilde{A}(z, \omega),
$$

which leads us to identify $\tilde{R}(\omega)$ with the electric susceptibility $\chi(\omega)$. From the Fourier transform of Eq. 2.5 one immediately derives the Kramers-Krönig relations of dispersion that connect the real and imaginary parts of $\chi(\omega)$, i.e. the index of refraction and the absorption coefficient. This is the expression of causality in the frequency domain. Specifically, the index of refraction is related to the absorption coefficient by:

$$
n(\omega)=1+\frac{c}{\omega_{0}} \frac{1}{2 \pi} \int \frac{\alpha\left(\omega^{\prime}\right)}{\omega-\omega^{\prime}} d t^{\prime}
$$

Then, Eq. 2.2 shall be changed accordingly into:

$$
A_{\text {out }}(\omega)=A_{\text {in }}(\omega) \exp (-\alpha(\omega) z / 2+i[n(\omega)-1] z) .
$$

In the small optical density limit, the response field given by Eq. 2.3 shall be complemented by $\mathrm{i} A_{\text {in }}(\omega)[n(\omega)-1] z$. One easily verifies that this additional contribution cancels the non-causal part of the pedestal.

At the line center $\omega_{0}, n\left(\omega_{0}\right)-1$ vanishes and one recovers Eq. 2.2. This result is apparently puzzling. Since $A\left(\omega_{0}\right)$ represents nothing but the pulse area, i.e. the time integral of the pulse envelope, Eq. 2.2 predicts that the pulse area vanishes at the absorbing medium output when 


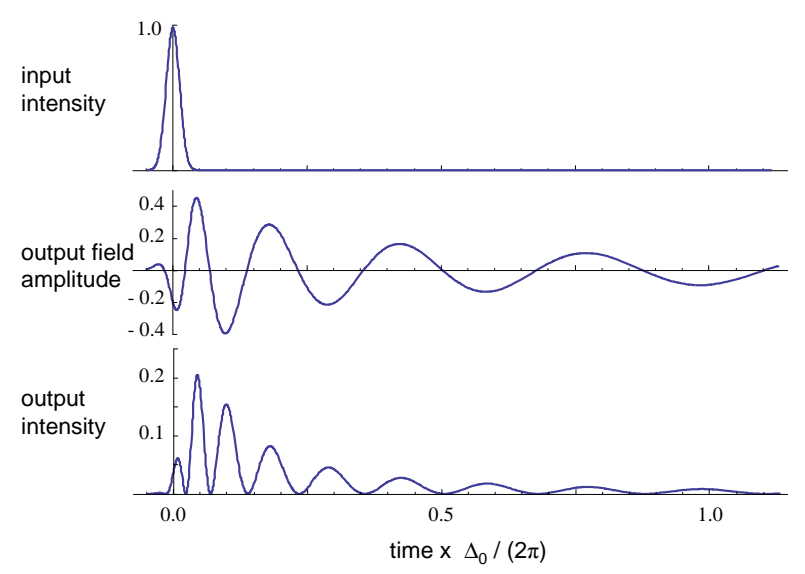

Figure 1: Distortion of a broadband short pulse by a $\Delta_{0}$-wide, absorption line. The pulse bandwidth is $\approx 40$ times larger than $\Delta_{0}$. The pulse spectrum is centered on the line. From top to bottom: input intensity, output field amplitude, output intensity. Output intensity and amplitude are scaled to the input. The field envelope oscillations make the vanishing of the pulse area consistent with the efficient transmission of energy.

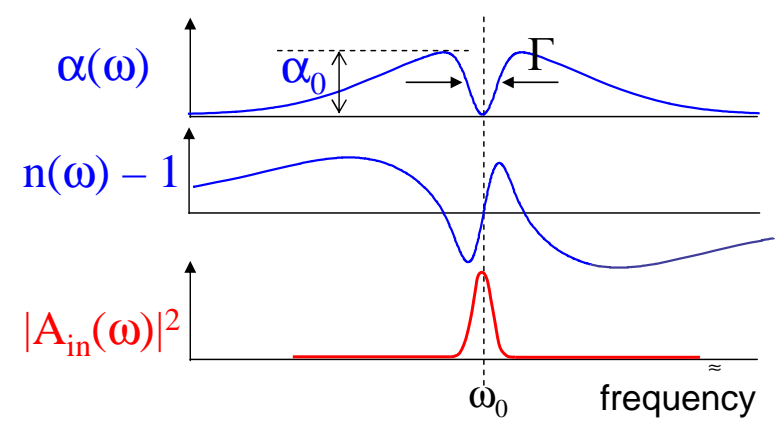

Figure 2: transparency window: correlated variations of $\alpha(\omega)$ and $n(\omega)$; relative width of the transparency window and the transmitted, slowed down, pulse.

$\alpha(\omega) z>>1$. This seems to be contradictory with the fact that, if the pulse bandwidth is large enough, all the electromagnetic energy should be transmitted without dissipation. As shown in Fig. 1, the effect of the phase term in Eq. 2.8, far away from the absorption band, solves this apparent contradiction $[1,2]$. Indeed this term makes the field envelope oscillate, giving rise to opposite sign contributions that make the vanishing of the pulse area consistent with the conservation of energy ${ }^{2}$.

\section{Slow light}

The absorption bandwidth reflects both the finite lifetime of the atomic superposition states and the width of the transition frequency distribution. The latter quantity, known as the inhomogeneous

\footnotetext{
${ }^{2}$ The restriction to coherent pulses, enacted as a rule in the introduction, can be circumvented in certain conditions. An incoherent emission can be considered as a train of contiguous and mutually independent sub-pulses whose duration equals the inverse spectral width of the incoherent emission. In Ref. [2] the coherent properties of incoherent light are detected by field cross-correlation of the transmitted light with a reference beam derived from the same source. This way one is able to discriminate the material response associated with each elementary sub-pulse.
} 
width, may result from the Doppler broadening caused by the atom translational motion. Let the bandwidth be dominated by the width of the transition frequency distribution. Then a $\Gamma$-wide transparency window may be burnt in this distribution at $\omega_{0}$, provided $\Gamma$ is larger than the inverse lifetime of the atomic superposition states. To practically burn a spectral hole, one can transfer the atoms to an auxilliary shelving state, over the $\Gamma$-wide interval. According to Eq. 2.7, the modified absorption profile is reflected in the spectral variations of the index of refraction, as illustrated in Fig. 2. A narrow-band light pulse, centered at $\omega_{0}$ and narrower than $\Gamma$, is exposed to a quasi-linear variation of the index of refraction, since the second derivative of $n(\omega)$ vanishes at the center of the symmetric window. The corresponding group velocity is given by:

$$
v_{g}=c /(1+\omega \mathrm{d} n(\omega) / \mathrm{d} \omega) \cong c /\left(1+\alpha_{0} c / \Gamma\right)
$$

where the absorption coefficient $\alpha_{0}$ represents the depth of the transparency window. A deep and narrow transparency window can reduce the pulse velocity by orders of magnitude [3]. As the pulse slows down, it undergoes a spatial compression. If $v_{g}$ is small enough, the pulse can be confined entirely within the boundaries of the absorbing medium. Its spatial extension is then $v_{g} / c$ times smaller within the material than in vacuum. Since the electromagnetic density of energy does not vary as light enters the medium, one concludes that the energy carried by the pulse is reduced by the same $v_{g} / c$ factor. This is quite surprising since the pulse is not absorbed, but only slowed down, and recovers all its initial energy at the exit of the material. It can be shown that the energy is stored within the off-resonant atoms, located on the sides of the transparency window [3,4]. Those atoms are excited in a superposition state that adiabatically follows the light pulse, propagating as a spin wave together with the pulse.

Further reduction of the group velocity hits against the difficulty of enlarging $\alpha_{0}$ and reducing $\Gamma$ simultaneously. Indeed $\alpha_{0}$ is the larger as the atoms interact more strongly with the field, but stronger interaction reduces the atomic superposition state lifetime, which enlarges the lower boundary to $\Gamma$. Electromagnetically induced transparency (EIT) circumvents this issue by the direct two-photon excitation of a long lifetime superposition state in the ground level of the optical transition. This way, the width of the transparency window is no longer controlled by $\Gamma$. With EIT, it was possible to slow light down to less than $20 \mathrm{~m} / \mathrm{s}$ [5]. The detailed description of this sophisticated technique, involving intense electromagnetic fields and three-level atoms, does not come within the scope of the present paper.

In the above discussion, light slowing down requires that the pulse spectrum fits within the transparency window. The linear absorption processing of broadband pulses has been considered recently in the context of quantum light storage $[6,7,8]$. Instead of burning a single hole within the absorption profile, one carves a periodic comb of absorbing teeth as illustrated in Fig. 3 . When the absorbing tooth width $\delta$ is much smaller than the $2 \pi / T$ spectral period, all the incoming energy is transmitted through the atomic frequency comb (AFC). However, all over the comb spectrum, the transmitted field undergoes a positive delay $L / v_{g}$, where $L$ represents the absorbing material depth. According to Eq. 3.1, the minimum group delay reads as $\tau_{0}=\langle\alpha\rangle L T / 4$, where $\langle\alpha\rangle=\alpha_{0} \delta T /(2 \pi)$ stands for the average absorption coefficient over a spectral period. No energy can exit the medium before time $T$ if $\tau_{0}>>T$. Since the delay is not uniform over the incoming pulse spectrum, the pulse is strongly distorted, being split into several components located at successive multiples of 


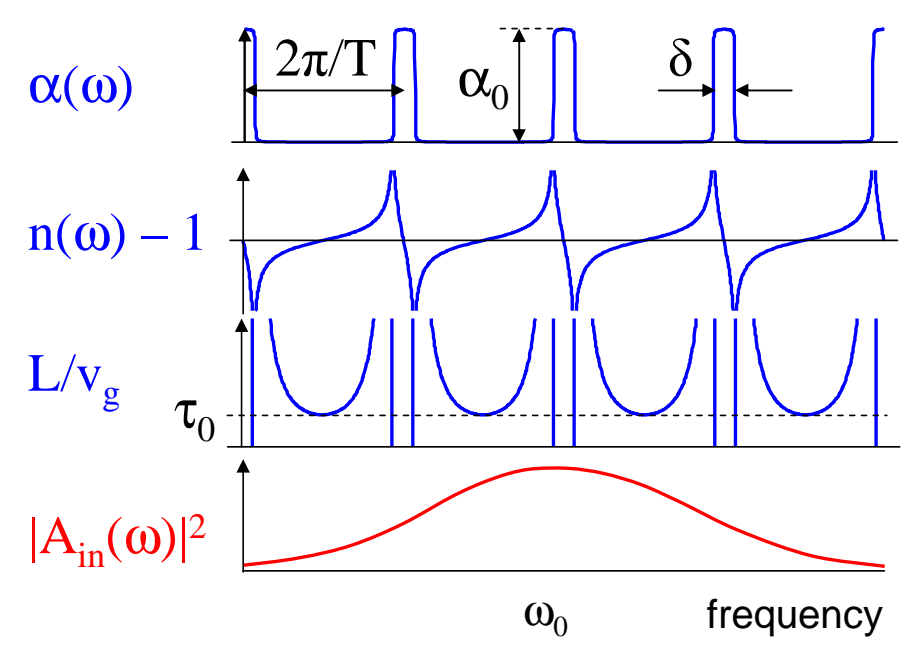

Figure 3: atomic frequency comb (AFC). A periodic comb of absorbing teeth gives rise to a periodic variation of $n(\omega)$. The resulting group delay $L / v_{g}$ is positive everywhere, with a minimum value at the middle of each transparency interval. The incoming pulse spectrum covers several teeth.

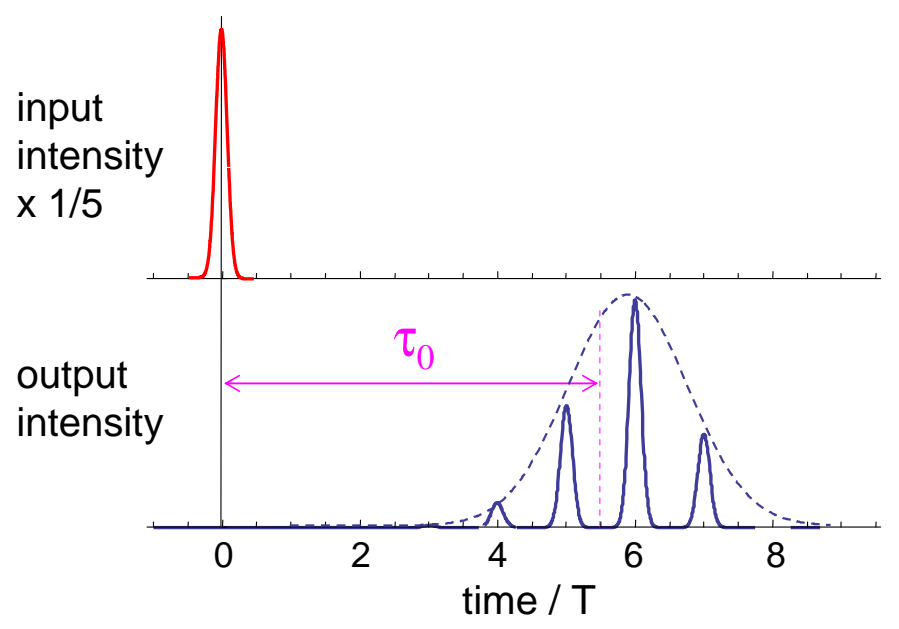

Figure 4: atomic frequency comb $(A F C)$ and slow light. A short incoming pulse, with a spectral width much larger than the $2 \pi / T$ period of the AFC, is delayed as it propagates through the AFC medium. We performed the displayed simulation by setting $\tau_{0}=\langle\alpha\rangle L T / 4=5 T$. The input pulse is split into several sub-pulses. Their overall envelope results from the stretching of the initial envelope.

$T$. Nevertheless, as shown in Fig. 4, the transmitted pulse envelope remains a smooth function that results from the stretching of the input profile.

\section{Conclusion}

Hopefully, the reader will agree that such a simple problem as the propagation of a weak planewave coherent pulse through an ensemble of two-level atoms can give rise to fascinating effects. Features such as multiple pulse generation and slow light are closely related to the combination of causality and the finite lifetime of atomic superposition states. However all these effects are 
revealed only in the time domain and depend on the pulse coherence. One may wonder whether such coherent pulses might be observed in astrophysics.

\section{References}

[1] Joshua E. Rothenberg, D. Grischkowsky, and A. C. Balant Phys. Rev. Lett. 53 (1984) 552.

[2] M. A. Bouchene, A. Débarre, J.-C. Keller, J.-L. Le Gouët, P. Tchénio, J. Opt. Soc. Am. 9 (1992) 281-89.

[3] R. Lauro, T. Chanelière, and J.-L. Le Gouët, Phys. Rev. A 79 (2009) 063844.

[4] D. Grischkowsky, Phys. Rev. A 7 (1973) 2096

[5] Lene Vestergaard Hau, S. E. Harris, Zachary Dutton, Cyrus H. Behroozi, Nature 397 (1999) 594-598

[6] Hugues de Riedmatten, Mikael Afzelius, Matthias Staudt, Christoph Simon, Nicolas Gisin, Nature 456 (2008) 773-777.

[7] Mikael Afzelius, Christoph Simon, Hugues de Riedmatten, and Nicolas Gisin, Phys. Rev. A 79 (2009) 052329.

[8] T. Chanelière, J. Ruggiero, M. Bonarota, M. Afzelius, J.-L. Le Gouët, New J. Phys. 12 (2010) 023025. 